\title{
Protocol for the development of the Master Chemical Mechanism, MCM v3 (Part B): tropospheric degradation of aromatic volatile organic compounds
}

\author{
M. E. Jenkin ${ }^{1}$, S. M. Saunders ${ }^{2,3}$, V. Wagner ${ }^{2}$, and M. J. Pilling ${ }^{2}$ \\ ${ }^{1}$ Department of Environmental Science and Technology, Imperial College, Silwood Park, Ascot, Berkshire, SL5 7PY, UK \\ ${ }^{2}$ School of Chemistry, University of Leeds, Leeds, LS2 9JT, UK \\ ${ }^{3}$ Disciplines of Chemistry and Geography, University of Western Australia, Nedlands, 6009 Western Australia
}

Received: 17 September 2002 - Published in Atmos. Chem. Phys. Discuss.: 7 November 2002

Revised: 27 January 2003 - Accepted: 27 January 2003 - Published: 12 February 2003

\begin{abstract}
Kinetic and mechanistic data relevant to the tropospheric degradation of aromatic volatile organic compounds (VOC) have been used to define a mechanism development protocol, which has been used to construct degradation schemes for 18 aromatic VOC as part of version 3 of the Master Chemical Mechanism (MCM v3). This is complementary to the treatment of 107 non-aromatic VOC, presented in a companion paper. The protocol is divided into a series of subsections describing initiation reactions, the degradation chemistry to first generation products via a number of competitive routes, and the further degradation of first and subsequent generation products. Emphasis is placed on describing where the treatment differs from that applied to the non-aromatic VOC. The protocol is based on work available in the open literature up to the beginning of 2001, and some other studies known by the authors which were under review at the time. Photochemical Ozone Creation Potentials (POCP) have been calculated for the 18 aromatic VOC in MCM v3 for idealised conditions appropriate to north-west Europe, using a photochemical trajectory model. The POCP values provide a measure of the relative ozone forming abilities of the VOC. These show distinct differences from POCP values calculated previously for the aromatics, using earlier versions of the MCM, and reasons for these differences are discussed.
\end{abstract}

\section{Introduction}

Aromatic hydrocarbons make a significant contribution to anthropogenic emissions of volatile organic compounds (VOC), representing a major component of vehicle exhaust

Correspondence to: M. E. Jenkin (m.jenkins@ic.ac.uk) and emissions from other combustion processes, and evaporative emissions of petroleum and from industrial processes and solvent usage (e.g. PORG, 1993; UK NAEI, 2002). It is well established that the gas phase degradation of VOC in general plays a central role in the generation of a variety of secondary pollutants, including ozone and secondary organic aerosol, SOA (e.g. Jenkin and Clemitshaw, 2000, and references therein). By virtue of their generally high reactivity and emissions, the oxidation of aromatic hydrocarbons is believed to make a substantial contribution to the formation of ozone on local and regional scales (Derwent et al., 1996; Calvert et al., 2002). In addition, their contribution to the formation of SOA in urban areas is believed to dominate (Odum et al., 1997), by virtue of their oxidation to products of comparatively low volatility in significant yield (Calvert et al., 2002).

The representation of mechanisms describing the degradation of aromatic hydrocarbons in air quality models has long been hampered by an incomplete knowledge of the detailed chemistry. As a result, laboratory experimental research in this field has been particularly active in recent years (e.g. Calvert et al., 2002, and references therein), and understanding of certain aspects of the degradation chemistry has improved. The available information has been used to define a mechanism development protocol for aromatic VOC, which is presented in the current paper. The protocol has been used to construct degradation schemes for 18 aromatic VOC as part of version 3 of the Master Chemical Mechanism (MCM v3). The treatment of 107 non-aromatic VOC is described separately in a companion paper (Saunders et al., 2003).

(C) European Geosciences Union 2003 


\section{Master Chemical Mechanism}

The MCM is a near-explicit chemical mechanism describing the detailed gas phase degradation of a series of emitted VOC, and the resultant generation of ozone and other secondary pollutants, under conditions appropriate to the planetary boundary layer. The philosophy behind the construction of the MCM is to use available information on the kinetics and products of elementary reactions relevant to VOC oxidation to build up a near-explicit representation of the degradation mechanisms, as described in detail for non-aromatic VOC in the companion paper (Saunders et al., 2003) and previously by Jenkin et al. (1997). In the absence of definitive published information on the elementary processes involved in aromatic degradation, provisional representations of the degradation of aromatic VOC were included in versions 1 and 2 of the MCM, as discussed further in Sect. 5. MCM v3 is the first version to contain a rigorous, consistent treatment of the degradation of aromatics at a level of detail comparable with that of other VOC classes (Jenkin et al., 1997; Saunders et al., 2003). The present paper contains a description of the construction methodology which forms the basis of the representation available in MCM v3: http://www.chem. leeds.ac.uk/Atmospheric/MCM/mcmproj.html The methodology aims to take into consideration work available in the open literature up to the beginning of 2001, and some other studies known by the authors which were under review at the time.

\section{Structure and methodology}

The protocol, outlined in Sect. 4, has been developed to allow the construction of comprehensive, consistent gas phase degradation schemes for aromatic VOC in MCM v3. The broad methodology follows that described in the companion paper for non-aromatic VOC (Saunders et al., 2003), such that a given organic compound is degraded into a set of 'first generation products', which are themselves further degraded within the same general framework. This process is continued until the chemistry either yields $\mathrm{CO}_{2}$, or until an organic product or radical is generated for which the subsequent chemistry is represented elsewhere in MCM v3. However, the detailed methodology applied to aromatic VOC (particularly for the initial oxidation sequence, and the further degradation of some first generation products) differs considerably from that described for non-aromatic VOC (Saunders et al., 2003).

In Sects. 4.1-4.3, the treatment of the initiation reactions with $\mathrm{OH}$ radicals and, where applicable, $\mathrm{O}_{3}$ and $\mathrm{NO}_{3}$ radicals, is described. Photolysis reactions, which are significant for aromatic aldehydes and a series of products of aromatic oxidation, are identified in Sect. 4.4. In Sect. 4.5, the degradation chemistry to first generation products via a number of competitive routes is summarised, and guidelines are estab- lished to indicate how their relative importance varies with structure. In Sect. 4.6, the further degradation of first, and subsequent generation products is discussed. In some cases, this treatment is based wholly or partially on the rules defined for the degradation of non-aromatic VOC and their products, as described in the corresponding protocols (Jenkin et al., 1997; Saunders et al., 2003). Similarly to the methodology described in the companion paper (Saunders et al., 2003), the aromatic protocol is designed to incorporate some strategic simplification in the degradation schemes generated, because fully explicit schemes for the $\mathrm{C}_{6}-\mathrm{C}_{11}$ compounds treated would otherwise be impracticably large.

Finally, 'Photochemical Ozone Creation Potentials' (POCP) calculated with MCM v3, implemented in a photochemical trajectory model, are presented in Sect. 5. The POCP values provide a measure of the relative ozone forming abilities of the 18 aromatic VOC for idealised conditions appropriate to north-west Europe. The observed trends are discussed, and the values are compared with those reported previously as calculated with MCM v1 (Derwent et al., 1998) and MCM v2 (Jenkin et al., 2000).

\section{Protocol for mechanism development}

\subsection{OH radical initiation reactions}

\subsubsection{Rates of initiation reactions}

Reaction with $\mathrm{OH}$ radicals represents the major atmospheric fate for aromatic hydrocarbons in general. Rate coefficients for the reactions of $\mathrm{OH}$ with a series of aromatic compounds have been reviewed extensively by Atkinson (1989; 1994) and very recently by Calvert et al. (2002), with recommendations made in a large number of cases. The recommendations of Calvert et al. (2002) allow rate coefficients to be assigned in all but two cases for the aromatic VOC in MCM v3, and these were applied accordingly (see Table 1). Where experimental data are unavailable, methods for estimating rate coefficients can be applied to aromatic compounds containing a variety of substituent groups (e.g. Kwok et al., 1995; Calvert et al., 2002). The available data demonstrate that methyl and ethyl substituents have almost identical activating influences on aromatic ring reactivity. Consequently, rate coefficients for the two species in MCM v3 for which data are unavailable (1-ethyl-3,5-dimethylbenzene and 1,3-diethyl-5methylbenzene) were taken to be identical to that of the trimethyl-substituted analogue, 1,3,5-trimethylbenzene.

\subsubsection{Initial radical products}

For aromatic hydrocarbons, the $\mathrm{OH}$ initiated chemistry proceeds mainly by addition to the aromatic ring to generate an $\mathrm{OH}$-aromatic adduct. For alkyl-substituted aromatic hydrocarbons, a small but significant fraction of the reaction proceeds by $\mathrm{H}$-atom abstraction from the alkyl side chain. 
Table 1. Rate coefficients for the reactions of $\mathrm{OH}$ and $\mathrm{NO}_{3}$ with aromatic VOC used in MCM v3

\begin{tabular}{lcc}
\hline VOC & $\mathbf{1 0}^{\mathbf{1 2}} k_{(\mathrm{OH})}$ & $\mathbf{1 0}^{\mathbf{1 6}} k_{\left(\mathrm{NO}_{3}\right)}$ \\
\hline & $\mathrm{cm}^{3}$ molecule-1 s & $\mathrm{cm}^{3}$ molecule $^{-1} \mathrm{~s}^{-1}$ \\
\hline benzene & $2.33 \exp (-193 / \mathrm{T})^{a}$ & - \\
toluene & $1.81 \exp (338 / \mathrm{T})^{a}$ & - \\
ethylbenzene & $7.0^{a}$ & $1.2^{a, d}$ \\
o-xylene & $13.6^{a}$ & $4.1^{a}$ \\
m-xylene & $23.1^{a}$ & $2.6^{a}$ \\
p-xylene & $14.3^{a}$ & $5^{a}$ \\
propylbenzene & $5.8^{a}$ & $1.4^{a, d}$ \\
i-propylbenzene & $6.3^{a}$ & $1.4^{a, d}$ \\
1,2,3,-trimethylbenzene & $32.7^{a}$ & $19^{a}$ \\
1,2,4,-trimethylbenzene & $32.5^{a}$ & $18^{a}$ \\
1,3,5,-trimethylbenzene & $56.7^{a}$ & $8.8^{a}$ \\
o-ethyltoluene & $11.9^{a}$ & $7.1^{a, c}$ \\
m-ethyltoluene & $18.6^{a}$ & $4.5^{a, c}$ \\
p-ethyltoluene & $11.8^{a}$ & $8.6^{e}$ \\
1-ethyl-3,5-dimethylbenzene & $56.7^{a, b}$ & $15^{a, c}$ \\
1,3-diethyl-5-methylbenzene & $56.7^{a, b}$ & $26^{a, c}$ \\
styrene & $58.0^{a}$ & $15000^{a}$ \\
benzaldehyde & $12.9^{a}$ & $24^{a}$ \\
\hline
\end{tabular}

$\underline{\text { Notes }}$

$\bar{a}$ Based on recommendation of Calvert et al. (2002); ${ }^{b}$ Assumed equivalent to 1,3,5-trimethylbenzene (see text); ${ }^{c}$ Ethyl substituent is assumed to activate the reactivity by a factor of 1.72 compared with a methyl group, based on a comparison of the reactivity of p-ethyltoluene and p-xylene. Rate coefficients for ethylbenzene, o-ethyltoluene, m-ethyltoluene, 3,5-dimethylethylbenzene and 1,3-diethylmethylbenzene thus inferred from data for toluene, o-xylene, m-xylene and 1,3,5-trimethylbenzene (Calvert et al., 2002); ${ }^{d}$ Propyl substituents are assumed to activate the reactivity by a factor of 2.0 compared with a methyl group, based on a comparison of the reactivity of p-i-propyltoluene and toluene. Rate coefficients for propylbenzene and i-propylbenzene thus inferred from data for toluene (Calvert et al., 2002); ${ }^{e}$ Based on relative rate data of Bolzacchini et al. (1999) as reported by Calvert et al. (2002).

Where the relative importance of abstraction and addition has been defined by laboratory kinetic or product investigations (e.g. as summarised by Atkinson, 1994, and Calvert et al., 2002) the data are used accordingly. In a small number of cases where information is unavailable, the relative importance was assumed to be equivalent to that of the closest analogue for which information is available. Because abstraction from the alkyl side chain is minor $(\leq 10 \%)$ in all cases considered, the subsequent chemistry is simplified by using a single representative radical in each case. Depending on the symmetry of the aromatic hydrocarbon, there can be up to six distinct addition points for the $\mathrm{OH}$ group, although available information indicates that the favourability of attack positions relative to an existing substitution varies considerably in the general order ortho- $>$ para- $>$ ipso- $>$ meta- (e.g. Andino et al., 1996). Furthermore, each resultant $\mathrm{OH}$-aromatic adduct (i.e. a hydroxycyclohexadienyl radical, with alkyl substitutions where appropriate) is resonancestabilised, such that subsequent reaction (e.g. with $\mathrm{O}_{2}$ ) can potentially take place at up to three positions. Consequently, even for comparatively simple aromatics, substantial complexity in the representation of the chemistry is possible in the initial reaction step. As described further in Sect. 4.5, the subsequent chemistry is propagated by the reactions of two representative hydroxy-aromatic adducts in each case. For the reactions of $\mathrm{OH}$ with styrene and benzaldehyde, addition of $\mathrm{OH}$ to the aromatic ring is minor (e.g. Atkinson, 1994; Calvert et al., 2002). In the case of styrene, attack is assumed to occur exclusively by addition to the vinyl substituent group, with formation of a single representative $\mathrm{OH}$ adduct. In the case of benzaldehyde, the mechanism proceeds by exclusive abstraction of the aldhehydic hydrogen, to generate the benzoyl radical.

\section{2 $\quad \mathrm{O}_{3}$ initiation reactions}

As discussed previously (Jenkin et al., 1997; Saunders et al., 2003), $\mathrm{O}_{3}$ initiated degradation chemistry is included for those VOC for which both the following relations apply:

$k\left(\mathrm{O}_{3}+\mathrm{VOC}\right)>10^{-8} k(\mathrm{OH}+\mathrm{VOC})$
$k\left(\mathrm{O}_{3}+\mathrm{VOC}\right)>10^{-19} \mathrm{~cm}^{3}$ molecule ${ }^{-1} \mathrm{~s}^{-1}$

On this basis, only the reaction of styrene with $\mathrm{O}_{3}$ is sufficiently rapid to be considered for the aromatic VOC in 


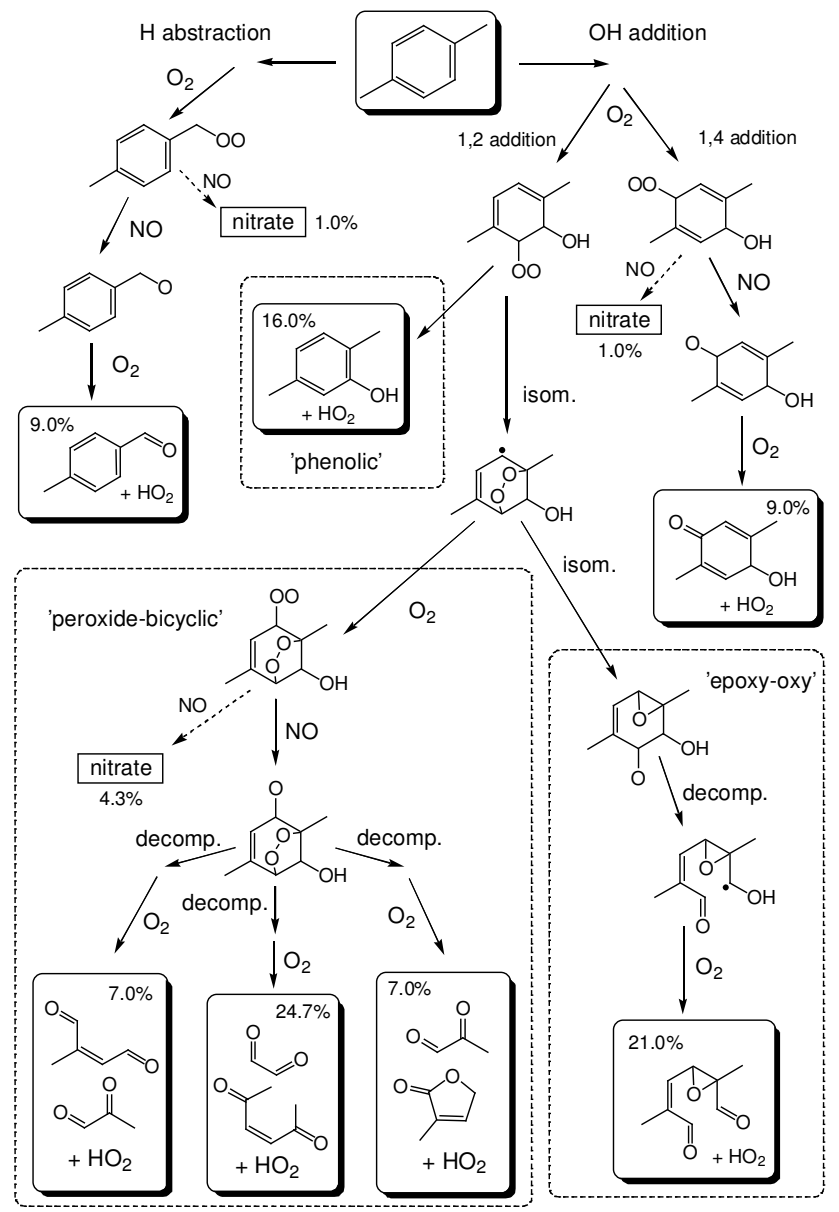

Fig. 1. Partial schematic representation of the $\mathrm{OH}$-initiated oxidation of p-xylene to first generation products, as treated in MCM v3. The chemistry shown assumes that $\mathrm{RO}_{2}$ radicals (with the exception of that formed initially from 1,2 addition of $\mathrm{OH}$ and $\mathrm{O}_{2}$ ) react exclusively with NO. The first generation products are shown in boxes, and the associated percentage yields are appropriate to these conditions.

MCM v3. The assigned rate coefficient is based on the recommendation of Calvert et al. (2002). The subsequent scheme development follows the methodology described in the previous protocols (Jenkin et al., 1997; Saunders et al., 2003), with the major aromatic product, benzaldehyde, degraded as described elsewhere in the present paper.

\section{3 $\quad \mathrm{NO}_{3}$ radical initiation reactions}

As discussed previously (Jenkin et al., 1997; Saunders et al., 2003), $\mathrm{NO}_{3}$ initiated degradation chemistry is included for those VOC for which both the following relations apply:

$k\left(\mathrm{NO}_{3}+\mathrm{VOC}\right)>10^{-5} k(\mathrm{OH}+\mathrm{VOC})$

$k\left(\mathrm{NO}_{3}+\mathrm{VOC}\right)>10^{-16} \mathrm{~cm}^{3}$ molecule $\mathrm{e}^{-1} \mathrm{~s}^{-1}$

$\mathrm{NO}_{3}$ initiated degradation is therefore considered for all the aromatic VOC in MCM v3, with the exception of benzene and toluene which are excluded on the basis of the second criterion. Reaction with $\mathrm{NO}_{3}$ potentially makes a significant contribution to the removal of styrene, but is a minor sink in the majority of cases.

\subsubsection{Rates of initiation reactions}

Rate coefficients for the reactions of $\mathrm{NO}_{3}$ with a series of aromatic compounds have been reviewed by Atkinson (1991, 1994), Wayne et al. (1991) and very recently by Calvert et al. (2002). The review of Calvert et al. (2002) makes recommendations, or presents data, which can be applied in 9 of the 16 cases for which $\mathrm{NO}_{3}$ initiated chemistry is treated. In the other cases, rate coefficients are estimated on the basis of the available data, as indicated in Table 1.

\subsubsection{Initial radical products}

The reactions of $\mathrm{NO}_{3}$ with alkyl-substituted aromatic hydrocarbons proceed via net abstraction of hydrogen from the alkyl group (e.g. Calvert et al., 2002). These reactions are assumed to yield the same initial radical generated from the $\mathrm{OH}$ abstraction reaction (Sect. 4.1.2). The reaction with benzaldehyde proceeds via abstraction of the aldehydic $\mathrm{H}$-atom, leading to the production of the benzoyl radical. The attack of $\mathrm{NO}_{3}$ on styrene is assumed to proceed by exclusive addition to the vinyl substituent, leading to the formation of a nitrooxy-substituted radical.

\subsection{Initiation by photolysis}

Initiation by photolysis is considered for benzaldehyde, and also for many other complex carbonyl compounds, hydroperoxides and organic nitrates which are generated as degradation products of the complete series of aromatic VOC. As described previously (Jenkin et al., 1997; Saunders et al., 2003), the methodology used in the MCM involves assigning photolysis parameters to a core number of reactions for which absorption cross section and quantum yield data are available. Some of these parameters are also used to define the photolysis rates of a much larger number of related species, for which the required information is not available. With the exception of the unsaturated dicarbonyl products discussed below in Sect. 4.6.3, the existing methodology (Saunders et al., 2003) is applied to the aromatic systems in MCM v3.

\subsection{Mechanisms to first generation products}

The dominant reaction under tropospheric conditions for all the organic radicals generated from the initiation reactions described above is assumed to be with oxygen, leading initially to the formation of a variety of peroxy radicals, $\mathrm{RO}_{2}$ :

$\mathrm{R}+\mathrm{O}_{2}(+\mathrm{M}) \rightarrow \mathrm{RO}_{2}(+\mathrm{M})$ 
Table 2. Relative branching ratios assigned to $\mathrm{OH}$-initiated oxidation routes to first generation products ${ }^{a}$

\begin{tabular}{|c|c|c|c|c|c|}
\hline \multirow[t]{2}{*}{ Hydrocarbon } & \multirow{2}{*}{$\begin{array}{c}\mathbf{H} \\
\text { abstraction }\end{array}$} & \multirow{2}{*}{$\begin{array}{c}1,4 \mathrm{OH} \\
\text { addition }\end{array}$} & \multicolumn{3}{|c|}{ 1,2 $\mathrm{OH}$ addition } \\
\hline & & & Phenolic & Peroxide-bicyclic & Epoxy-oxy ${ }^{c}$ \\
\hline benzene $^{d}$ & - & 0.10 & 0.25 & 0.33 & 0.33 \\
\hline toluene & 0.10 & 0.10 & 0.25 & 0.33 & 0.22 \\
\hline ethylbenzene $^{e}$ & 0.07 & 0.10 & 0.26 & 0.34 & 0.23 \\
\hline o-xylene & 0.05 & 0.10 & 0.16 & 0.55 & 0.14 \\
\hline m-xylene & 0.04 & 0.10 & 0.17 & 0.50 & 0.19 \\
\hline p-xylene & 0.10 & 0.10 & 0.16 & 0.43 & 0.21 \\
\hline propylbenzene ${ }^{e}$ & 0.07 & 0.10 & 0.26 & 0.34 & 0.23 \\
\hline i-propylbenzene ${ }^{e}$ & 0.07 & 0.10 & 0.26 & 0.34 & 0.23 \\
\hline $1,2,3$,-trimethylbenzene & 0.06 & 0.10 & 0.03 & 0.70 & 0.11 \\
\hline $1,2,4$,-trimethylbenzene & 0.06 & 0.10 & 0.03 & 0.61 & 0.20 \\
\hline 1,3,5,-trimethylbenzene & 0.03 & 0.10 & 0.04 & 0.79 & 0.04 \\
\hline o-ethyltoluene $f$ & 0.05 & 0.10 & 0.16 & 0.55 & 0.14 \\
\hline m-ethyltoluene ${ }^{f}$ & 0.04 & 0.10 & 0.17 & 0.50 & 0.19 \\
\hline p-ethyltoluene $f$ & 0.10 & 0.10 & 0.16 & 0.43 & 0.21 \\
\hline 1-ethyl-3,5-dimethylbenzene $g$ & 0.03 & 0.10 & 0.04 & 0.79 & 0.04 \\
\hline 1,3-diethyl-5-methylbenzene $g$ & 0.03 & 0.10 & 0.04 & 0.79 & 0.04 \\
\hline
\end{tabular}

Notes

$\bar{a}$ Ratios assigned where possible on the basis of reported product yields of hydroxyarenes, aromatic aldehydes and $\alpha$-dicarbonyls in $\mathrm{NO}_{\mathrm{x}}-$ present systems as summarised by Calvert et al. (2002). ${ }^{b} 1,4$ addition assumed to be $10 \%$ in all systems (see text); ${ }^{c}$ Epoxy-oxy route generally assigned balance of flux; ${ }^{d}$ Phenol yield based on reported data. Balance of flux divided equally between peroxide-bicyclic and epoxy-oxy routes; ${ }^{e}$ ratios of 1,2 addition routes based on those for toluene; ${ }^{f}$ based on data for corresponding xylene; ${ }^{g}$ based on data for 1,3,5-trimethylbenzene.

The distribution of these radicals from the $\mathrm{OH}$-initiated chemistry, as represented in MCM v3, is shown in Table 2 for benzene and the aromatic hydrocarbons with alkyl substituents. As indicated in Sect. 4.1.2, the yields of those generated from hydrogen atom abstraction from the alkyl groups are consistently $\leq 10 \%$. The sequential addition of $\mathrm{OH}$ and $\mathrm{O}_{2}$ to the aromatic ring is assumed to generate two types of hydroxycyclohexadienyl peroxy radical (with alkyl substituents, where appropriate) in each case, to represent ' 1,2 ' and ' 1,4' addition. The latter are significantly less stable (owing to the loss of conjugation), but are included to provide a minor route to para-quinone products, which have been observed in some systems (e.g. Seuwen and Warneck, 1996; Berndt et al., 1999; Smith et al., 1999). These are therefore assigned a low probability of $10 \%$ in each case, with those formed from 1,2 addition being the dominant peroxy radical generated in each of the systems.

The main features of the subsequent mechanisms leading to first generation products are shown schematically in Fig. 1, using p-xylene as an example. The details of these mechanisms are described in the following paragraphs.

\subsection{1 $\mathrm{RO}_{2}$ radicals from $\mathrm{H}$-abstraction}

The minor $\mathrm{RO}_{2}$ radicals generated from $\mathrm{H}$ abstraction from alkyl substituents are assumed to react as conventional $\mathrm{RO}_{2}$ radicals, and are therefore treated as described by in the companion paper (Saunders et al., 2003), such that reactions with $\mathrm{NO}, \mathrm{NO}_{3}, \mathrm{HO}_{2}$ and $\mathrm{RO}_{2}$ radicals in general are treated. The terminating channels of these reactions generate a variety of nitrate, hydroperoxide, carbonyl and alcohol products. The propagating channels, most significantly the major channel of the reaction with $\mathrm{NO}$, yield the corresponding oxy radicals (RO):

$\mathrm{RO}_{2}+\mathrm{NO} \rightarrow \mathrm{RO}+\mathrm{NO}_{2}$

The sole fate of the $\mathrm{RO}$ radicals is reaction with $\mathrm{O}_{2}$, leading to the well-established formation of an aromatic aldehyde and $\mathrm{HO}_{2}$ (Calvert et al., 2002), e.g. p-tolualdehyde in the case of p-xylene (see Fig. 1). For aromatics containing alkyl groups which are not identical (e.g. 1,2,4-trimethylbenzene), a single $\mathrm{RO}_{2}$ radical is used to represent all such radicals formed following $\mathrm{H}$ atom abstraction, and the aromatic aldehyde product is therefore also used as a single representative. 


\subsection{2 $\mathrm{RO}_{2}$ radicals from 1,4 addition of $\mathrm{OH}$ and $\mathrm{O}_{2}$}

On the basis of studies of allyl and substituted-allyl peroxy radicals (e.g. Jenkin et al., 1993, 1998), the minor 1,4-hydroxycyclohexadienyl type $\mathrm{RO}_{2}$ radicals are also assumed to react as conventional $\mathrm{RO}_{2}$ radicals, and are therefore treated as described by Saunders et al. (2003). The subsequently-formed RO radicals are assumed to react exclusively with $\mathrm{O}_{2}$, by analogy with the alloxy radical (Jenkin et al., 1993). As shown in Fig. 1 for p-xylene, this generates $\mathrm{HO}_{2}$ and a cyclic hydroxycarbonyldiene product.

\subsection{3 $\mathrm{RO}_{2}$ radicals from 1,2 addition of $\mathrm{OH}$ and $\mathrm{O}_{2}$}

Published information is consistent with the major $\mathrm{RO}_{2}$ radicals formed from 1,2 addition of $\mathrm{OH}$ and $\mathrm{O}_{2}$ undergoing rapid unimolecular rearrangements (e.g. Lay et al., 1996; Ghigo and Tonachini, 1999; Bohn and Zetzsch, 1999; Bohn, 2001), such that the conventional reactions of $\mathrm{RO}_{2}$ radicals cannot compete under most tropospheric conditions. On the basis of available product information (e.g. Calvert et al., 2002), three generic reaction channels are assigned to the 1,2-hydroxycyclohexadienyl type $\mathrm{RO}_{2}$ radicals in MCM v3:

(i) Isomerisation and decomposition to form a hydroxyarene and $\mathrm{HO}_{2}$ (denoted the 'phenolic' route in Table 2 and Fig. 1): This route is used to describe the formation of the well-established hydroxyarene products such as phenol from benzene, and the cresol isomers from toluene (Calvert et al., 2002). In each case, a single representative hydroxyarene product is generated (e.g. ocresol is used to represent the three isomers). It is noted that these products may alternatively be generated from the initial $\mathrm{OH}$ adduct by a direct reaction with $\mathrm{O}_{2}$ (e.g. Lay et al., 1996; Ghigo and Tonachini, 1998). However, the overall chemistry of these representations is indistinguishable, such that the representation employed is consistent with either mechanism.

(ii) Ring closure to form a peroxide bridge (denoted the 'peroxide-bicyclic' route in Table 2 and Fig. 1): This route, based on that proposed by Atkinson et al. (1980), is used to describe the formation of the well-established $\alpha$-dicarbonyl products, such as glyoxal and methylglyoxal, as first generation products. Reported information indicates that $\alpha$-dicarbonyl products are generated from the oxidation of all simple mono-aromatics studied to date (Calvert et al., 2002), and the observations of Bethel et al. (2000) and Volkamer et al. (2001) are consistent with their prompt formation as first generation products.

As shown for p-xylene in Fig. 1, the chemistry in each system is propagated by a single series of radicals. Addition of $\mathrm{O}_{2}$ leads to the formation of a complex bicyclic $\mathrm{RO}_{2}$ radical, which is assumed to undergo the conventional reactions with $\mathrm{NO}, \mathrm{NO}_{3}, \mathrm{HO}_{2}$ and $\mathrm{RO}_{2}$ radicals in general (Saunders et al., 2003), and the corresponding formation of minor nitrate, hydroperoxide, carbonyl and alcohol products. The subsequently-formed RO radical decomposes by a ring-opening mechanism to form $\mathrm{HO}_{2}$, an $\alpha$-dicarbonyl product and a further co-product (e.g. see Atkinson et al., 1980; Smith et al., 1999; Calvert et al., 2002). On the basis of reported product data, the co-product is either an $\alpha, \beta$-unsaturated$\gamma$-dicarbonyl (e.g. butenedial; 4-oxo-2-pentenal), or a corresponding cyclic furanone product where formation is possible (Smith et al., 1999). Although the product channels strictly available for the representative bicyclic $\mathrm{RO}$ radical are limited, the $\mathrm{RO}$ radical in a given system is assigned several decomposition channels to allow formation of all possible $\alpha$-dicarbonyl products and combinations of co-product which could be formed from isomeric forms of the radical. The relative yields assigned to the $\alpha$-dicarbonyls, following RO radical decomposition in the various aromatic systems, are summarised in Table 3, based on consensus yields from reported data (Bethel et al., 2000; Calvert et al., 2002). Thus, in the case of p-xylene, for example (Fig. 1), routes to the formation of either glyoxal or methylglyoxal are represented, in the ratio $0.64 / 0.36$. The sole organic coproduct for glyoxal is 3-hexene-2,5-dione (since the positioning of the methyl groups precludes formation of a corresponding $\mathrm{C}_{6}$ furanone product), whereas the overall formation of methylglyoxal is divided into two equal routes forming 2-methylbutenedial and a $\mathrm{C}_{5}$ furanone as co-products.

(iii) Isomerisation to form an cyclic epoxy-oxy radical (denoted the 'epoxy-oxy' route in Table 2 and Fig. 1): As shown in Fig. 1, the epoxy-oxy intermediate may be formed from the further rearrangement of the peroxidebicyclic intermediate discussed above. This route is based on the qualitative observations of Yu and Jeffries (1997) and Kwok et al. (1997), and is included to represent the balance of the $\mathrm{OH}$-initiated chemistry not accounted for by the routes discussed above. Consistent with the rules presented previously (Jenkin et al., 1997; Saunders et al., 2003) for oxy radicals containing a $\beta$ hydroxy group, the epoxy-oxy radical undergoes ringopening, followed by reaction with $\mathrm{O}_{2}$ to generate $\mathrm{HO}_{2}$ and a representative epoxydicarbonylene product.

\subsubsection{Branching ratios}

As indicated above, the relative importance of the various reaction channels presented in Tables 2 and 3 is based, where possible, on consensus yields for the aromatic aldehyde, hydroxyarene and $\alpha$-dicarbonyl products (e.g. as summarised by Calvert et al., 2002). It should be noted that, even for these well-established products, there is considerable variation in the reported yields, and that even for the most stud- 
Table 3. Relative yields of $\alpha$-dicarbonyl products assigned to aromatic systems ${ }^{a}$

\begin{tabular}{lccc}
\hline & $\mathrm{HC}(\mathrm{O}) \mathrm{CHO}$ & $\mathrm{RC}(\mathrm{O}) \mathrm{CHO}^{b}$ & $\mathrm{RC}(\mathrm{O}) \mathrm{C}(\mathrm{O}) \mathrm{R}^{b}$ \\
\hline benzene $^{c}$ & 1.00 & - & - \\
alkylbenzene $^{b}$ & 0.50 & 0.50 & - \\
o-dialkylbenzene $^{\text {m-dialkybenzene }}$ & 0.16 & 0.49 & 0.35 \\
p-dialkylbenzene $^{\text {1,2,3-trialkylbenzene }}$ & 0.22 & 0.78 & - \\
1,2,4-trialkylbenzene $^{c}$ & 0.64 & 0.36 & - \\
1,3,5-trialkylbenzene $^{c}$ & 0.10 & 0.26 & 0.64 \\
\hline
\end{tabular}

$\underline{\text { Notes }}$

${ }^{a}$ Ratios assigned on the basis of reported relative product yields of glyoxal, methylglyoxal and biacetyl from toluene, xylenes and trimethylbenzenes as summarised by Calvert et al. (2002); ${ }^{b}$ For aromatics containing combinations of different alkyl groups, contribution is subdivided pro rata; ${ }^{c}$ Only one $\alpha$-dicarbonyl possible by definition.

ied systems, only ca. $50 \%$ of the aromatic loss has been accounted for by observed products. In particular, the reported yields of the $\alpha$-dicarbonyl co-products $(\alpha, \beta$-unsaturated$\gamma$-dicarbonyls and furanones) tend to be significantly lower than those of the $\alpha$-dicarbonyls themselves, indicating that knowledge of the detailed chemistry of the initial oxidation step is still incomplete. Nevertheless, the methodology described above is believed to provide an adequate representation of current understanding.

\subsection{5 $\mathrm{RO}_{2}$ radicals from styrene and benzaldehyde}

The reactions of the $\mathrm{RO}_{2}$ radicals generated from the $\mathrm{OH}$ and $\mathrm{NO}_{3}$ initiated degradation of styrene, and the subsequent chemistry to first generation products, are represented by the methodology described in detail previously (Saunders et al., 2003). The chemistry applicable to the further degradation of benzaldehyde (and other aromatic aldehydes generated as products of aromatic oxidation) is presented below in Sect. 4.6.2.

\subsection{Reactions of degradation products}

The degradation of aromatic VOC by the reactions summarised in the preceding subsections leads to a wide variety of first generation oxygenated products of varying complexity, which are themselves further degraded in the MCM. Broadly speaking, the products may be divided into two groups: (i) those which retain the aromatic ring, and (ii) those which are formed following ring opening processes. Many of those in the second category are degraded in MCM v3 by the methodology described for non-aromatic VOC (Jenkin et al., 1997; Saunders et al., 2003), as indeed are the majority of subsequent generation products. The present section is therefore limited to a description of those products for which the previous protocols are not fully applied.

\subsubsection{Hydroxyarenes}

The degradation of the hydroxyarenes is initiated by reactions with $\mathrm{OH}$ and $\mathrm{NO}_{3}$. Rate coefficients have been recommended for the reactions of $\mathrm{OH}$ with phenol, cresol isomers, dimethylphenol isomers and selected trimethylphenol isomers (Calvert et al., 2002), and these values are applied accordingly. In the limited number of cases where kinetics information is unavailable, the assigned rate coefficient is based on that for the closest analogue for which information is available. Rate coefficients for the reactions of $\mathrm{NO}_{3}$ with phenol and the cresol isomers have also been recommended (Calvert et al., 2002). For the larger hydroxyarenes, the rate coefficient for the closest cresol analogue is applied.

Reported information is consistent with the reactions proceeding via an addition mechanism, with subsequent partial elimination of phenoxy radicals (alkyl substituted where appropriate) and $\mathrm{H}_{2} \mathrm{O}$ or $\mathrm{HNO}_{3}$ (e.g. Atkinson, 1994). Kinetics studies of phenoxy radicals indicate that reactions with $\mathrm{NO}, \mathrm{NO}_{2}$ and $\mathrm{O}_{3}$ are likely to be competitive under ambient conditions (Platz et al., 1998; Berho et al., 1998; Tao and Lee, 1999). However, in MCM v3, a given phenoxy-type radical is generally assumed to react exclusively with $\mathrm{NO}_{2}$ to generate a representative 1-hydroxy-2-nitroarene (e.g. 2nitrophenol from the reaction of the phenoxy radical itself):

$\mathrm{C}_{6} \mathrm{H}_{5} \mathrm{O}+\mathrm{NO}_{2} \rightarrow \mathrm{HOC}_{6} \mathrm{H}_{4} \mathrm{NO}_{2}$

This is justified because the reaction with NO is reversible on a comparatively short timescale (ca. 1 min: Berho et al., 1998), and the reaction with $\mathrm{O}_{3}$ is likely to regenerate phenoxy radicals, predominantly as part of the following null cycle:

$\mathrm{C}_{6} \mathrm{H}_{5} \mathrm{O}+\mathrm{O}_{3} \rightarrow \mathrm{C}_{6} \mathrm{H}_{5} \mathrm{O}_{2}+\mathrm{O}_{2}$

$\mathrm{C}_{6} \mathrm{H}_{5} \mathrm{O}_{2}+\mathrm{NO} \rightarrow \mathrm{C}_{6} \mathrm{H}_{5} \mathrm{O}+\mathrm{NO}_{2}$ 
$\mathrm{NO}_{2}+\mathrm{h} v\left(+\mathrm{O}_{2}\right) \rightarrow \mathrm{NO}+\mathrm{O}_{3}$

The yield of the phenoxy-type radicals from the initiation reactions is therefore based on the yields of the hydroxynitroarene products reported by Atkinson et al. (1992) for $\mathrm{OH}$ and $\mathrm{NO}_{3}$-initiated oxidation of phenol and the cresol isomers. For the larger hydroxyarenes, the yields are assumed to be the same as for the closest cresol analogue. In the specific cases of the 2,4,6-trialkyl substituted phenoxy-type radicals generated in the 1,3,5-trimethylbenzene, 1-ethyl-3,5dimethylbenzene and 1,3-diethyl-5-methylbenzene systems, reaction with $\mathrm{NO}_{2}$ to form 1-hydroxy-2-nitroarene products is precluded by the presence of the adjacent alkyl groups. In these cases, the phenoxy-type radicals are assumed to regenerate the corresponding hydroxyarene by a slow reaction with $\mathrm{HO}_{2}$.

In the absence of reported mechanistic information, the balance of the reaction (i.e. not forming phenoxy-type radicals) in each case is assumed to follow a mechanism analogous to the peroxide-bicyclic route described above, with product channels limited to the formation of one $\alpha$ dicarbonyl product and a corresponding hydroxyl- or nitrooxy-substituted $\alpha, \beta$-unsaturated- $\gamma$-dicarbonyl co-product. These products are degraded by the general methodology described by Saunders et al. (2003).

The hydroxynitroarenes are further degraded by reaction with $\mathrm{OH}$, with the kinetics of the reactions involving nitrophenol and nitrocresols based on the data summarised by Atkinson (1994). For other hydroxynitroarenes, the rate coefficient is assumed to be equivalent to that of the closest nitrocresol analogue. In the absence of mechanistic information, the degradation mechanisms are simplified such that ring-opening occurs to generate products already present in MCM v3. These mechanisms are broadly based on the peroxide-bicyclic route described above.

\subsubsection{Aromatic aldehydes}

In addition to removal by photolysis (see Sect. 4.4), the degradation of the aromatic aldehydes is initiated by reactions with $\mathrm{OH}$ and $\mathrm{NO}_{3}$. Rate coefficients have been recommended for the reactions of $\mathrm{OH}$ with benzaldehyde and dimethylbenzaldehyde isomers (Calvert et al., 2002), and these values are applied accordingly. In other cases, rate coefficients are estimated using the structure-activity relationship (SAR) method of Kwok and Atkinson (1995) and Atkinson (2000). Kinetic data are available for the reaction of $\mathrm{NO}_{3}$ with benzaldehyde (Calvert et al., 2002). For other aromatic aldehydes, a generic rate coefficient for reactions of $\mathrm{NO}_{3}$ with aldehydes is applied in MCM v3, the value of which is close to that reported for benzaldehyde (Jenkin et al., 1997; Saunders et al., 2003).

On the basis of reported studies for benzaldehyde (e.g. Noziere et al., 1994; Caralp et al., 1999; Calvert et al., 2002), the reactions are assumed to lead to overall abstraction of the aldehydic hydrogen atom, followed by addition of $\mathrm{O}_{2}$, leading to the production of aromatic acyl peroxy radicals, $\Phi$ $\mathrm{C}(\mathrm{O}) \mathrm{O}_{2}$ (where $\Phi$ is a phenyl or an alkyl-substituted phenyl group):

$$
\begin{aligned}
& \Phi-\mathrm{CHO}+\mathrm{OH}\left(+\mathrm{O}_{2}\right) \rightarrow \Phi-\mathrm{C}(\mathrm{O}) \mathrm{O}_{2}+\mathrm{H}_{2} \mathrm{O} \\
& \Phi-\mathrm{CHO}+\mathrm{NO}_{3}\left(+\mathrm{O}_{2}\right) \rightarrow \Phi-\mathrm{C}(\mathrm{O}) \mathrm{O}_{2}+\mathrm{HNO}_{3}
\end{aligned}
$$

The $\Phi-\mathrm{C}(\mathrm{O}) \mathrm{O}_{2}$ radicals are treated as described previously for conventional acyl peroxy radicals (Jenkin et al., 1997; Saunders et al., 2003), undergoing reactions with $\mathrm{NO}, \mathrm{NO}_{2}$, $\mathrm{NO}_{3}, \mathrm{HO}_{2}$ and $\mathrm{RO}_{2}$ radicals in general. This is fully consistent with reported information on the reactions of benzoyl peroxy radicals, formed from benzaldehyde (e.g. Lightfoot et al., 1992; Noziere et al., 1994; Caralp et al., 1999). Similarly, the subsequently-formed aromatic acyl-oxy radicals are assumed to decompose to yield $\mathrm{CO}_{2}$ and phenyl type radicals (Caralp et al., 1999), followed by addition of $\mathrm{O}_{2}$ to yield phenyl peroxy radicals, $\mathrm{F}-\mathrm{O}_{2}$ :

$\Phi-\mathrm{C}(\mathrm{O}) \mathrm{O}\left(+\mathrm{O}_{2}\right) \rightarrow \Phi-\mathrm{O}_{2}+\mathrm{CO}_{2}$

The $\Phi-\mathrm{O}_{2}$ radicals also undergo a conventional set of reactions (Saunders et al., 2003), with the resultant phenoxy type radicals reacting exclusively with $\mathrm{NO}_{2}$ to form nitrophenol products as described in the previous section.

\subsection{3 $\alpha, \beta$-unsaturated- $\gamma$-dicarbonyls}

The degradation of the $\alpha, \beta$-unsaturated- $\gamma$-dicarbonyls is initiated by reaction with $\mathrm{OH}, \mathrm{O}_{3}$ and $\mathrm{NO}_{3}$, and by photolysis. Rate coefficients have been determined for the reactions of $\mathrm{OH}$ with butenedial, 4-oxo-2-pentenal and 3-hexene-2,5dione (Tuazon et al., 1985; Bierbach et al., 1994; Calvert et al., 2002), and these values are applied accordingly. In other cases, rate coefficients are estimated using the method described previously for unsaturated carbonyls (Jenkin et al., 1997), with this methodology also being used to infer the relative importance of addition to the double bond and abstraction of $\mathrm{H}$ from the aldehyde group (where applicable).

The mechanism following $\mathrm{OH}$ addition to the double bond follows the standard non-aromatic chemistry described by Jenkin et al. (1997) and Saunders et al. (2003). The chemistry following abstraction of $\mathrm{H}$ from the aldehyde group also largely follows the conventional methodology, but with incorporation of an additional route to generate a furandione product in appropriate cases, as has been observed in laboratory studies of the oxidation of $\alpha, \beta$-unsaturated $\gamma$ dicarbonyls (Bierbach et al., 1994), and also as products of aromatic hydrocarbon oxidation (e.g. Forstner et al., 1997). This is achieved by inclusion of a cyclisation reaction for the intermediate acyl-oxy radicals, which is assumed to compete equally with the standard decomposition reaction, e.g.: 
Table 4. Generic rate coefficients used for the reactions of $\mathrm{O}_{3}$ with $\alpha, \beta$-unsaturated- $\gamma$-dicarbonyls in MCM v3

\begin{tabular}{lrc}
\hline $\begin{array}{l}\text { generic } \\
\alpha, \beta \text {-unsaturated- } \gamma \text {-dicarbonyl }\end{array}$ & $k(298)^{b}$ & Comment \\
\hline $\mathrm{RC}(\mathrm{O}) \mathrm{CH}=\mathrm{CHCHO}$ & & \\
$\mathrm{RC}(\mathrm{O}) \mathrm{C}\left(\mathrm{R}^{\prime}\right)=\mathrm{CHCHO}$ & 2.0 & $\mathrm{c}$ \\
$\mathrm{RC}(\mathrm{O}) \mathrm{C}\left(\mathrm{R}^{\prime}\right)=\mathrm{C}\left(\mathrm{R}^{\prime}\right) \mathrm{CHO}$ & 14.0 & $\mathrm{c}$ \\
$\mathrm{R}^{\prime} \mathrm{C}(\mathrm{O}) \mathrm{CH}=\mathrm{CHC}(\mathrm{O}) \mathrm{R}^{\prime}$ & 5.0 & $\mathrm{~d}$ \\
$\mathrm{R}^{\prime} \mathrm{C}(\mathrm{O}) \mathrm{C}\left(\mathrm{R}^{\prime}\right)=\mathrm{CHC}(\mathrm{O}) \mathrm{R}^{\prime}$ & 12.5 & $\mathrm{e}$ \\
$\mathrm{R}^{\prime} \mathrm{C}(\mathrm{O}) \mathrm{C}\left(\mathrm{R}^{\prime}\right)=\mathrm{C}\left(\mathrm{R}^{\prime}\right) \mathrm{C}(\mathrm{O}) \mathrm{R}^{\prime}$ & 35.0 & $\mathrm{e}$ \\
\hline
\end{tabular}

$\underline{\text { Notes }}$

${ }^{a} \mathrm{R}=\mathrm{H}$ or alkyl, $\mathrm{R} '=$ alkyl $;^{b}$ Units $10^{-1} 8 \mathrm{~cm}^{3}$ molecule ${ }^{-1} \mathrm{~s}^{-1}$;

${ }^{c}$ Based on method of Jenkin et al. (1997). Rate coefficient is mean of those calculated for $\mathrm{R}=\mathrm{H}$ and $\mathrm{R}=$ alkyl; ${ }^{d}$ Mean of reported measurements for cis- and trans-3-hexene-2,5-dione (Tuazon et al., 1985); ${ }^{e}$ Estimated relative to mean of reported measurements for cis- and trans-3-hexene-2,5-dione (Tuazon et al., 1985), assuming activating influence of additional R' groups based on method of Jenkin et al. (1997).

$\mathrm{HC}(\mathrm{O}) \mathrm{CH}=\mathrm{CHC}(\mathrm{O}) \mathrm{O} \rightarrow \mathrm{HC}(\mathrm{O}) \mathrm{CH}=\mathrm{CH}+\mathrm{CO}_{2}$<smiles>O=C1C=CC(O)O1</smiles><smiles>O=C1C=CC(=O)O1</smiles>

Reported information on the kinetics of the reactions of $\alpha$, $\beta$-unsaturated- $\gamma$-dicarbonyls with $\mathrm{O}_{3}$ is limited to one study of the reaction with 3-hexene-2,5-dione isomers (Tuazon et al., 1985; Calvert et al., 2002). Rate coefficients for a series of generic species were therefore estimated on the basis of that study, and the estimation methodology described previously (Jenkin et al., 1997). The resultant parameters (shown in Table 4) are also consistent with the estimates of Bierbach et al. (1994). The mechanism following initiation by reaction with $\mathrm{O}_{3}$ follows the methodology described in detail previously (Jenkin et al., 1997).

There have been no studies of the reactions of $\mathrm{NO}_{3}$ with $\alpha, \beta$-unsaturated- $\gamma$-dicarbonyls, although kinetics information is available for the reactions of $\mathrm{NO}_{3}$ with the $\mathrm{C}_{4} \alpha$, $\beta$-unsaturated aldehydes, crotonaldehyde and methacrolein (Atkinson et al., 1988; Kwok et al., 1996). Comparison of the rate coefficients of these reactions with those available for $\mathrm{C}_{4}$ aldehydes (see summary in Table 1 of Saunders et al., 2003) suggest that the reactivity of the unsaturated aldehydes is approximately a factor of two lower than that of the corresponding saturated aldehyde. This rule (in conjunction with the substantial database on $\mathrm{NO}_{3}+$ aldehyde reactions) was used, therefore, to infer rate coefficients for the reactions of $\mathrm{NO}_{3}$ with $\alpha, \beta$-unsaturated- $\gamma$-dicarbonyls containing aldehyde groups. In these cases, reaction was assumed to occur exclusively at the aldehyde group(s). Support for this assumption is provided by the observed low reactivity of $\mathrm{NO}_{3}$ radicals with the $\alpha, \beta$-unsaturated ketone, methylvinyl ketone (Calvert et al., 2000), suggesting that addition to a double bond adjacent to a carbonyl group is unfavoured. Accordingly, $\alpha, \beta$-unsaturated- $\gamma$-diketones (e.g. 3-hexene-2,5dione) were assumed not to react with $\mathrm{NO}_{3}$.

Chamber studies have demonstrated that the photolyis of $\alpha, \beta$-unsaturated- $\gamma$-dicarbonyls is particularly rapid (Bierbach et al., 1994), and may represent the dominant atmospheric sink (Volkamer et al., 2001). The generic rate coefficients applied to the photolysis reactions of aldehydes and ketones (Saunders et al., 2003) are therefore inappropriate. On the basis of the observations for a series of $\alpha$, $\beta$-unsaturated- $\gamma$-dicarbonyls (Bierbach et al., 1994), generic photolysis rates of $5.0 \times 10^{-2} \mathrm{~J}_{\mathrm{NO}_{2}}$ were applied to species containing aldehyde groups, and $2.8 \times 10^{-2} \mathrm{~J}_{\mathrm{NO}_{2}}$ to $\alpha, \beta$ unsaturated- $\gamma$-diketones. These rates are approximately $40 \%$ of the observed decay rates, and are used to represent the sum of the radical forming channels alone, e.g.

$$
\mathrm{RC}(\mathrm{O}) \mathrm{CH}=\mathrm{CHCHO} \rightarrow \mathrm{RC}(\mathrm{O}) \mathrm{CH}=\mathrm{CHC}(\mathrm{O})+\mathrm{H}
$$

$$
\rightarrow \mathrm{RC}(\mathrm{O}) \mathrm{CH}=\mathrm{CH}+\mathrm{HCO}
$$

$$
\rightarrow \mathrm{RCO}+\mathrm{HC}(\mathrm{O}) \mathrm{CH}=\mathrm{CH}
$$

with each channel assigned an equivalent fraction of the total.

\subsubsection{Epoxydicarbonylenes}

As indicated above (Sect. 4.5.3) the epoxy-oxy route leading to the generation of representative epoxydicarbonylene products was included to account for the balance of the $\mathrm{OH}$ initiated reaction of the aromatic hydrocarbons not accounted for by the other routes. Although such epoxy products have been detected (Yu and Jeffries, 1997), their yields have not been quantified and there is little information on their degradation chemistry. Because of their multifunctional structure, initiation reactions with $\mathrm{OH}, \mathrm{O}_{3}, \mathrm{NO}_{3}$ and direct photolysis are treated in $\mathrm{MCM} \vee 3$. For $\mathrm{OH}$ initiation, an estimate of the overall rate coefficient is made using structure activity relationships (Kwok and Atkinson 1995; Atkinson, 2000). For the reactions with $\mathrm{NO}_{3}$ and $\mathrm{O}_{3}$, and for photolysis, estimates of the rates are made by analogy with the $\alpha, \beta$-unsaturated $-\gamma$ dicarbonyls, as these are the most structurally similar compounds in the mechanism. The initial distribution of radicals, the subsequent chemistry and the further degradation of products follows the methodology described in the previous protocols (Jenkin et al. 1997; Saunders et al., 2003). 


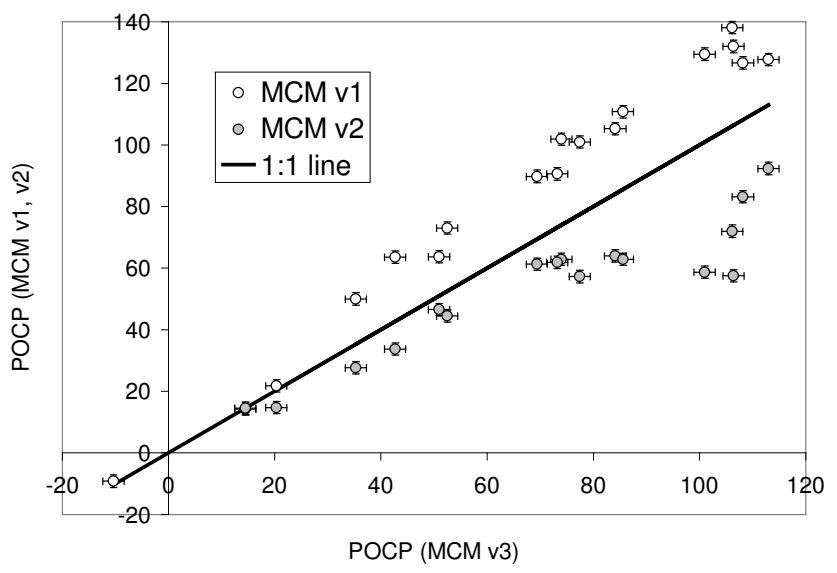

Fig. 2. Comparison of POCP values calculated for aromatic VOC using MCM v3, and those reported previously using MCM v1 (Derwent et al., 1998) and MCM v2 (Jenkin et al., 2000).

\section{Photochemical Ozone Creation Potentials (POCP)}

The Photochemical Ozone Creation Potential (POCP) concept was initially developed to describe the relative abilities of organic compounds to generate ozone on a regional scale over north-west Europe (Derwent and Jenkin, 1991), and the MCM has previously been applied to calculation of POCP values (e.g. Derwent et al., 1998). As described in detail in the companion paper (Saunders et al., 2003), POCP values are calculated from the impact of small changes in the emissions of individual VOC on simulated ozone formation, using a Photochemical Trajectory Model (PTM) operating on an idealised multi-day trajectory over north-west Europe. POCP values are defined relative to a value of 100 for ethene, and represent the quantity of ozone formed from unit mass emission of a given VOC, relative to that from emission of an identical mass of ethene.

POCP values were calculated for all 124 non-methane VOC using MCM v3. The results for the 106 non-aromatic VOC are presented and discussed in the companion paper (Saunders et al., 2003). The POCP values calculated for the 18 aromatic VOC are presented in Table 5, and are compared with those calculated with MCM v1 (Derwent et al., 1998) and MCM v2 (Jenkin et al., 2000) in Fig. 2. Unlike the situation for the majority of non-aromatic VOC discussed in the companion paper (Saunders et al., 2003), there are distinct differences between the POCP values reported previously for the aromatics, and those calculated with MCM v3. This arises because of the substantial mechanistic differences between the mechanisms.

The MCM v1 schemes are generally the most efficient at generating ozone, and the MCM v2 schemes the least efficient. As indicated in Sect. 2, these versions included provisional representations of the degradation of aromatic VOC. In MCM v1, the degradation chemistry essentially followed the ring opening route proposed by Atkinson et al. (1980) (referred to above as the 'peroxide-bicyclic' mechanism), with one set of $\alpha$-dicarbonyl and $\alpha, \beta$-unsaturated- $\gamma$-dicarbonyl products formed in each case. The degradation of the latter products was also highly simplified. Sensitivity studies have shown that this route is particularly efficient at generating ozone (Jenkin et al., 2000), owing to a combination of two NO-to- $\mathrm{NO}_{2}$ conversions occurring in the initial oxidation step, and the prompt formation of species (e.g. $\alpha-$ dicarbonyls) which can photolyse efficiently to generate radicals, thereby promoting ozone formation from VOC in general. In MCM v2, more detailed schemes were developed to explore a proposed degradation route involving the formation of 'aromatic oxide/oxepin' intermediates (Klotz et al., 1997, 1998). As shown in Fig. 2, these schemes are much less efficient at generating ozone. This is partly because only one NO-to- $\mathrm{NO}_{2}$ conversion occurs in the initial oxidation step, and because the yield of species which can photolyse efficiently to generate radicals is lower, and their formation is delayed to the third oxidation step.

With the exception of the least reactive aromatic hydrocarbon, benzene (POCP $\approx 20$ ), the POCP values calculated with MCM v3 lie consistently lower than those calculated with MCM v1 by between 10 and 30\%, and greater than those calculated with MCM v2 to varying degrees. The monoalkyl-substituted aromatic hydrocarbons are calculated to have comparatively low POCP values (in the range 3553), whereas those for the dialkyl- and trialkyl species are significantly higher, lying in the ranges 69-84 and 101113 , respectively. The latter two sets of species therefore have POCP values comparable with those calculated for the alkenes (Saunders et al., 2003), making them among the most potent ozone generators. As discussed previously (Derwent et al., 1998; Jenkin and Hayman, 1999), the observed trend in POCP values results from a combination of kinetic and structural factors. For the less reactive VOC, the POCP scale broadly correlates with that of $\mathrm{OH}$ reactivity (see Table 1), with the impacts of structural differences superimposed on the trend. For the more reactive VOC (with $k_{(\mathrm{OH})} \gg 10^{-11} \mathrm{~cm}^{3}$ molecule ${ }^{-1} \mathrm{~s}^{-1}$ ) further increases in reactivity have little effect on POCP, but the influences of structural differences are more apparent. For example, 1,3-5-trimethylbenzene has a significantly higher $\mathrm{OH}$ reactivity than the other trimethylbenzenes, but it has the lowest POCP value. This arises because the other isomers (particularly 1,2,3-trimethylbenzene) generate biacetyl, $\mathrm{CH}_{3} \mathrm{C}(\mathrm{O}) \mathrm{C}(\mathrm{O}) \mathrm{CH}_{3}$, in significant yield (see Table 3). Owing to its low reactivity with $\mathrm{OH}$ radicals, biacetyl is almost exclusively photolysed to generate radicals. Its formation therefore has a greater impact on ozone generation than that of the other $\alpha$-dicarbonyls, for which removal by $\mathrm{OH}$ reaction is competitive. The influence of biacetyl formation is also apparent from a comparison of the POCP values for the xylene isomers.

Despite their high reactivity, the POCP values calculated 
Table 5. Photochemical Ozone Creation Potentials (POCP) calculated for 18 aromatic VOC in MCM v3 ${ }^{a}$

\begin{tabular}{lrlr}
\hline VOC & POCP & VOC & POCP \\
\hline ethene (reference VOC) & 100.0 & & \\
benzene & 20.3 & 1,2,4,-trimethylbenzene & 113.0 \\
toluene & 51.0 & 1,3,5,-trimethylbenzene & 106.2 \\
ethylbenzene & 52.5 & o-ethyltoluene & 69.4 \\
o-xylene & 84.1 & m-ethyltoluene & 74.0 \\
m-xylene & 85.6 & p-ethyltoluene & 73.2 \\
p-xylene & 77.5 & 1-ethyl-3,5-dimethylbenzene & 106.4 \\
propylbenzene & 42.7 & 1,3-diethyl-5-methylbenzene & 101.0 \\
i-propylbenzene & 35.3 & styrene & 14.5 \\
1,2,3,-trimethylbenzene & 108.2 & benzaldehyde & -10.4 \\
\hline
\end{tabular}

$\underline{\text { Notes }}$

${ }^{a}$ POCP values are quoted to one decimal place, not as an indication of inherent precision, but to facilitate comparisons. The precision in an individual POCP value is estimated to be \pm 2 POCP units.

for styrene and benzaldehyde are the lowest of all the aromatic VOC, in agreement with previous studies (Derwent et al., 1996, 1998). As indicated in Sect. 4.6.2, the degradation of benzaldehyde leads to the formation of nitrophenol in high yield. Nitrophenol is a comparatively unreactive reservoir for both free radicals and $\mathrm{NO}_{\mathrm{X}}$, and its formation therefore inhibits ozone formation, as reflected in the negative POCP value. The low POCP value for styrene arises because of its partial degradation to benzaldehyde.

\section{Conclusions}

The mechanism construction protocol described above in Sect. 4 has allowed consistent degradation schemes to be defined for 18 aromatic compounds as part of MCM v3. The methodology is based on reported information, and aims to take account of work which was in the public domain at the beginning of 2001. Although there are still clearly gaps and uncertainties in the understanding of aromatic degradation chemistry, this methodology is believed to provide a sound basis for ongoing mechanism development work, as described by Wagner et al. (2003).

Particular areas where understanding is lacking have been discussed in detail by Calvert et al. (2002). These include uncertainties in the mechanisms of the initial oxidation sequence(s) to first generation products, and limitations in the available information on the subsequent chemistry of many of the classes of product known to be generated. The degradation chemistry of first and subsequent generation products is believed to be particularly important in accounting for secondary radical generation and removal of $\mathrm{NO}_{\mathrm{X}}$ in chamber experiments (Wagner et al., 2003). This is therefore a main focus of ongoing work, facilitated by the constant emergence of new relevant data (e.g. Volkamer, 2002; Olariu et al., 2002; EXACT, 2002). In addition, a representation of gas-aerosol partitioning of the oxidised products of aromatic degradation is being developed to assist interpretation of the formation of secondary organic aerosol observed in aromatic systems (e.g. Odum et al., 1997; Forstner et al., 1997; EXACT, 2002).

Acknowledgements. The work described in this paper was funded by the UK Department for Environment, Food and Rural Affairs, DEFRA (under contracts EPG 1/3/70 and 1/3/143), and by the EU (as part of the EXACT project EVK-1999-00053). MEJ also acknowledges the UK Natural Environment Research Council, NERC, for support via a Senior Research Fellowship (NER/K/S/2000/00870). Thanks are due to Dick Derwent (UK Meteorological Office) for helpful discussions on the POCP calculations presented in Sect. 5. This work has also benefited from collaboration within the EUROTRAC-2 Chemical Mechanism Development (CMD) subproject.

\section{References}

Andino J. M., Smith J. N., Flagan R. C., Goddard W. A., and Seinfeld, J. H.: Mechanism of atmospheric photooxidation of aromatics: a theoretical study, J. Phys. Chem., 100, 10 967-10 980, 1996.

Atkinson, R.: Kinetics and mechanisms of the gas-phase reactions of the hydroxyl radical with organic compounds. J. Phys. Chem. Ref. Data, Monograph 1, 1989.

Atkinson, R.: Kinetics and mechanisms of the gas-phase reactions of the nitrate radical with organic compounds, J. Phys. Chem. Ref. Data, 20, 459-507, 1991.

Atkinson, R.: Gas-phase tropospheric chemistry of organic compounds, J. Phys. Chem. Ref. Data, Monograph 2, 1994.

Atkinson, R.: Atmospheric Oxidation. Contribution to "Handbook of Property Estimation Methods for Chemicals: Environmental and Health Sciences", R. S. Boethling and D. Mackay, CRC Press, 2000.

Atkinson, R., Carter, W.P.L., Darnall, K. R., Winer, A. M., Pitts, Jr., J. N.: A smog chamber and modelling study of the gas phase 
$\mathrm{NO}_{\mathrm{x}}$-Air photooxidation of toluene and the cresols, Int. J. Chem. Kinetics, 12, 779-834, 1980.

Berho, F., Caralp, F., Rayez, M. T., Lesclaux, R., and Ratajczak, E.: Kinetics and thermochemistry of the reversible combination reaction of the phenoxy radical with NO, J. Phys. Chem. A, 102, $1-8,1998$.

Berndt, T., Böge, O., and Hermann, H.: On the formation of benzene oxide/oxepin in the gas phase reaction of $\mathrm{OH}$ radicals with benzene, Chem. Phys. Lett., 314, 435-442, 1999.

Bethel H. L., Atkinson, R., and Arey, J.: Products of the gas-phase reactions of $\mathrm{OH}$ radicals with p-xylene and 1,2,3- and 1,2,4trimethylbenzene: effect of $\mathrm{NO}_{2}$ concentration, J. Phys. Chem. A, 104, 8922-8929, 2000.

Bierbach, A., Barnes, I., Becker, K. H., and Wiesen, E.: Atmospheric Chemistry of unsaturated carbonyls: butendial, 4-oxo2-pentenal, 3-hexene-2,5-dione, maleic-anhydride, 3H-furan-2one, and 5-methyl-3H-furan-2-one, Environ. Sci. Tech., 28 (4), 715-729, 1994.

Bohn, B.: Formation of peroxy radicals from $\mathrm{OH}$-toluene adducts and $\mathrm{O}_{2}$. J. Phys. Chem. A, 105, 6092-6101, 2001.

Bohn, B. and Zetzsch, C.: Gas phase reaction of the OH-benzene adduct with $\mathrm{O}_{2}$ : reversibility and secondary formation of $\mathrm{HO}_{2}$, Phys. Chem. Chem. Phys., 1, 5097-5107, 1999.

Calvert, J. G., Atkinson, R., Kerr, J. A., Madronich, S., Moortgat, G. K., Wallington, T. J., and Yarwood, G.: The mechanisms of atmospheric oxidation of alkenes, Oxford University Press, New York, ISBN 0-19-513177-0, 2000.

Calvert, J. G., Atkinson, R., Becker, K. H., Kamens, R. M., Seinfeld, J. H., Wallington, T. J., and Yarwood, G.: The mechanisms of atmospheric oxidation of aromatic hydrocarbons, Oxford University Press, New York, ISBN 0-19-514628-X, 2002.

Caralp, F., Foucher, V., Lesclaux, R., Wallington, T. J., and Hurley, M. D.: Atmospheric chemistry of benzaldehyde: UV absorption spectrum and reaction kinetics and mechanisms of the $\mathrm{C}_{6} \mathrm{H}_{5} \mathrm{C}(\mathrm{O}) \mathrm{O}_{2}$ radical, Phys. Chem. Chem. Phys., 1, 3509-3517, 1999.

Derwent R. G., Jenkin, M. E., and Saunders, S. M.: Photochemical Ozone Creation Potentials for a Large Number of Reactive Hydrocarbons Under European Conditions, Atmos. Environ., 30, 181-199, 1996.

Derwent, R. G., Jenkin, M. E., Saunders, S. M., and Pilling, M. J.: Photochemical ozone creation potentials for organic compounds in North West Europe calculated with a master chemical mechanism, Atmos. Environ., 32, 2429-2441, 1998.

EXACT: Second annual report on the EU project "Effects of the Oxidation of Aromatic Compounds in the Troposphere" (see http: //www.york.ac.uk/depts/eeem/exact1/ for further details), 2002.

Forstner, H. J. L., Flagan, S. C., and Seinfeld, J. H.: Secondary organic aerosol from the photooxidation of aromatic hydrocarbons: molecular composition, Environ. Sci. Tech., 31, 13451358, 1997.

Ghigo, G. and Tonachini, G.: Benzene oxidation in the troposphere: theoretical investigation on the possible competition of three postulated reaction channels, J. Amer. Chem. Soc., 120, 6753-6757, 1998.

Ghigo, G. and Tonachini, G.: From benzene to muconaldehyde: theoretical investigation of some tropospheric oxidation channels, J. Amer. Chem. Soc., 121, 8366-8372, 1999.

Jenkin, M. E. and Hayman, G. D.: Photochemical ozone creation potentials for oxygenated volatile organic compounds: sensitivity to variations in kinetic and mechanistic parameters, Atmos. Environ., 33, 1275-1293, 1999.

Jenkin, M. E. and Clemitshaw, K. C.: Ozone and other secondary photochemical pollutants: chemical processes governing their formation in the planetary boundary layer, Atmos. Environ., 34, 2499-2527, 2000.

Jenkin, M. E., Murrells, T. P., Shalliker, S. J., and Hayman, G. D.: Kinetics and product study of the self-reactions of allyl and allyl peroxy radicals at 296K, J. Chem. Soc. Faraday Transactions, 89, 433-446, 1993.

Jenkin, M. E., Saunders, S. M., and Pilling, M. J.: The tropospheric degradation of volatile organic compounds: a protocol for mechanism development, Atmos. Environ., 31, 81-104, 1997.

Jenkin, M. E., Boyd, A. A., and Lesclaux, R.: Peroxy radical kinetics resulting from the $\mathrm{OH}$-initiated oxidation of 1,3-butadiene, 2,3-dimethyl-1,3-butadiene and isoprene, J. Atmos. Chem., 29, 267-298, 1998.

Jenkin, M. E., Saunders, S. M., and Derwent, R. G.: Photochemical Ozone Creation Potentials for Aromatic Hydrocarbons: Sensitivity to Variations in Kinetic and Mechanistic Parameters, Proceedings of the conference Chemical Behaviour of Aromatic Hydrocarbons in the Troposphere, (Ed) Becker, K. H., Valencia, Spain, February 27-29, 2000, ISSN 1436-2198, 81-87, 2000.

Kwok, E.S.C. and Atkinson, R.: Estimation of hydroxyl radical reaction rate constants for gas-phase organic compounds using a structure-reactivity relationship: an update, Atmos. Environ., 29, 1685-1695, 1995.

Kwok, E. S. C., Aschmann, S. M., Akinson, R., and Arey, J.: Products of the gas-phase reactions of o-, m- and p-xylene with the $\mathrm{OH}$ radical in the presence and absence of $\mathrm{NO}_{X}$, J. Chem. Soc., Faraday Transactions, 93(16), 2847-2854, 1997.

Lay, T. H., Bozzelli, J. W., and Seinfeld, J. H.: Atmospheric photochemical oxidation of benzene: benzene $+\mathrm{OH}$ and the benzene-OH adduct (hydroxyl-2,4-cyclohexadienyl) $+\mathrm{O}_{2}$, J. Phys. Chem. A, 100, 6543-6554, 1996.

Odum, J. R., Jungkamp, T. P. W., Griffin, R. J., Flagan, R. C., and Seinfeld, J. H.: The atmospheric aerosol forming potential of whole gasoline vapor, Science, 276, 96-99, 1997.

Olariu, R. I., Barnes, I., Becker, K. H., Klotz, B., and Mocanu, R.: FT-IR study of the ring-retaining products from the reaction of $\mathrm{OH}$ radicals with phenol, o-, m-, p-cresol, Atmos. Environ., 36, 3685-3697, 2002.

Platz, J., Nielsen, O. J., Wallington, T. J., Ball, J. C., Hurley, M. D., Straccia, A. M., Schneider, W. F., and Sehested, J.: Atmospheric chemistry of the phenoxy radical, J. Phys. Chem. A, 102, 7964 7974, 1998.

PORG: Ozone in the United Kingdom, Third report of the UK Photochemical Oxidants Review Group, Department of the Environment, London, 1993.

Saunders, S. M., Jenkin, M. E., Derwent, R. G., and Pilling, M. J.: Protocol for the development of the Master Chemical Mechanism, MCM v3 (Part A): tropospheric degradation of nonaromatic volatile organic compounds, Atmos. Chem. Phys., 3, 161-180, 2003.

Seuwen, R. and Warneck, P.: Oxidation of toluene in NOX-free air: product distribution and mechanism, Int. J. Chem. Kinetics, 28, 315-332, 1996.

Smith, D. F., Kleindienst, T. E., and McIver, C. D.: Primary prod- 
uct distributions from the reaction of $\mathrm{OH}$ with m-, p-xylene, 1,2,4- and 1,3,5-trimethyl benzene, J. Atmos. Chem., 34, 339$364,1999$.

Tao, Z. and Li, Z:: A kinetics study on reactions of $\mathrm{C}_{6} \mathrm{H}_{5} \mathrm{O}$ with $\mathrm{C}_{6} \mathrm{H}_{5} \mathrm{O}$ and $\mathrm{O}_{3}$ at $298 \mathrm{~K}$, Int. J. Chem. Kinetics, 31, 65-72, 1999.

Tuazon, E. C., Atkinson, R., and Carter, W.P. L.: Atmospheric chemistry of cis- and trans-3-hexene-2,5-dione, Environ. Sci. Tech., 19, 265-269, 1985.

UK NAEI: The UK National Atmospheric Emissions Inventory (available at http://www.aeat.co.uk/netcen/airqual), 2002.

Volkamer, R., Platt, U., and Wirtz, K.: Primary and secondary glyoxal formation from aromatics: experimental evidence for the bicycloalkyl-radical pathway from benzene, toluene and pxylene, J. Phys. Chem. A, 105, 7865-7874, 2001.

Volkamer, R., Klotz, B., Barnes, I., Imamura, T., Wirtz, K.,
Washida, N., Becker, K. H., and Platt, U.: OH-initiated oxidation of benzene - Part I. Phenol formation under atmospheric conditions, Phys. Chem. Chem. Phys., 4 (9), 1598-1610, 2002.

Wagner, V., Jenkin, M. E., Saunders, S. M., Stanton, J., Wirtz, K., and Pilling, M. J.: Modelling of the Photooxidation of Toluene: Conceptual Ideas for Validating Detailed Mechanisms, Atmos. Chem. Phys., 3, 89-106, 2003.

Wayne, R. P., Barnes, I., Biggs, P., Burrows, J. P., Canos-Mas, C. E., Hjorth, J., LeBras, G., Moortgat, G. K., Perner, D., Poulet, G., Restelli, G., and Sidebottom, H.: The nitrate radical: physics, chemistry and the atmosphere, Atmos. Environ., 25A, 1-206, 1991.

Yu, J. Z. and Jeffries, H. E.: Atmospheric photooxidation of alkylbenzenes 2. Evidence for formation of epoxide intermediates, Atmos. Environ., 31 (15), 2281-2287, 1997. 